\title{
Efectos psicosociales de la enfermedad periodontal en la calidad de vida de pacientes de la Facultad de Odontologia (UdelaR) Un estudio cuali-cuantitativo.
}

\author{
Psychosocial effects of periodontal disease on the quality \\ of life of patients of the School of Dentistry (UdelaR) \\ A qualitative-quantitative study.
}

Efeitos psicossociais da doença periodontal na qualidade de vida

de pacientes da Faculdade de Odontologia (UdelaR)

Um estudo qualitativo-quantitativo

Alina Ariceta ${ }^{1}$ (D) 0000-0002-0513-8960
Luis Bueno $0^{2}$ (DD 0000-0002-7837-6492
Ernesto Andrade ${ }^{2}$ (D) 0000-0002-9511-3678

doi:10.22592/ode2021n37a1

Alejandra Arias $^{3}$

\section{Resumen}

Objetivos: Identificar los aspectos psicosociales de la enfermedad periodontal y su incidencia en la calidad de vida de las personas que la padecen.

Métodos: Se realizó un estudio mixto cuanti-cualitativo en pacientes de la facultad de odontología UdelaR. Los instrumentos utilizados fueron: entrevista (semiestructurada) con base en la teoría fundamentada y la aplicación del cuestionario OHIP-14 (Oral Health Impact Profile) que mide el grado de afectación en la calidad de vida (modelo teórico de Locker). Resultado: Se identifican los factores psicosociales que presenta esta población, así como la afectación emocional y a nivel social que provoca el diagnóstico de enfermedad periodontal. Los resultados muestran una afectación en la calidad de vida de la población de 1, 46 en una escala de 0-4, donde 4 es la máxima afectación. Las participantes femeninas mostraron mayor nivel de afectación en la calidad de vida $(1,54)$ en comparación con los hombres $(1$, 36). A mayor grado de instrucción más afectación en la calidad de vida.

Conclusiones: Existe una limitación en el enfoque biomédico en la atención de pacientes odontológicos, y por tanto la necesidad de realizar un abordaje integral en pacientes con enfermedad periodontal. Los profesionales odontólogos deben tener un enfoque biopsicosocial en la atención debido a la complejidad que presenta la enfermedad periodontal.

Palabras clave: calidad de vida, enfermedades periodontales, efecto biopsicosocial.

Departamento de Periodoncia. Universidad Católica del Uruguay. Montevideo, Uruguay aaricetaodontologa@gmail.com

Cátedra de Periodoncia. Facultad de Odontología. Universidad de la República, Uruguay

Instituto de Psicología de la Salud, Facultad de Psicología Universidad de la República, Uruguay 


\section{Abstract}

Objectives: To identify the psychosocial factors of periodontal disease and their impact on the quality of life of patients.

Methods: A mixed quantitative and qualitative study was conducted at the School of Dentistry, UdelaR. The instruments used were a semi-structured interview based on grounded theory and the application of the OHIP-14 (Oral Health Impact Profile) questionnaire that measures the degree of impairment of quality of life (Locker's theoretical model).

Results: This population's psychosocial factors are identified, as well as the emotional and social effects of periodontal disease diagnosis. The results show a 1.46 impact on people's quality of life on a scale of $0-4$, where 4 is the maximum impact. Women showed a higher level of impairment in quality of life (1.54) than men (1.36). The higher the educational level, the more the quality of life is affected.

Conclusions: The limitations of the biomedical approach to dental patient care and the need for a comprehensive approach in periodontal disease patients are clear. Dental professionals need a biopsychosocial care approach given the complexity of periodontal disease.

Keywords: quality of life, periodontal diseases, biopsychosocial impact.

\section{Resumo}

Objetivos: Identificar os aspectos psicossociais da doença periodontal e sua incidência na qualidade de vida das pessoas que sofrem com a doença.

Métodos: Foi realizado um estudo quantitativo e qualitativo misto. Os instrumentos utilizados foram: entrevista (semiestruturada), fundamentada na teoria fundamentada em dados, e aplicação do questionário OHIP-14 (Perfil de Impacto na Saúde Oral), que mede o grau de comprometimento da qualidade de vida (modelo teórico de Locker).

Resultado: são identificados os fatores psicossociais que essa população apresenta, bem como a afetação emocional e social que causa o diagnóstico de doença periodontal. Os resultados mostram uma afetação na qualidade de vida da população de 1, 46 em uma escala de 0-4, onde 4 é a afetaçáo máxima. As participantes do sexo feminino apresentaram maior nível de comprometimento da qualidade de vida $(1,54)$ em comparação aos homens (1, 36). Quanto maior o grau de escolaridade, mais a qualidade de vida será afetada.

Conclusóes: A limitação da abordagem biomédica no cuidado de pacientes odontológicos e a necessidade de realizar uma abordagem abrangente em pacientes com doença periodontal são evidentes. Os profissionais de odontologia devem ter uma abordagem biopsicossocial ao atendimento devido à complexidade da doença periodontal.

Palavras-chave: qualidade de vida, doenças periodontais, efeito biopsicossocial. 


\section{Introducción y antecedentes}

La periodontitis es una enfermedad inflamatoria crónica multifactorial asociada con el biofilm, se caracteriza por la destrucción progresiva del aparato de soporte dentario. Se manifiesta con pérdida de inserción clínica, reabsorción ósea, bolsa patológica y sangrado gingival. ${ }^{(1)}$

La enfermedad periodontal es una patología crónica que sufre el $90 \%$ de la población del mundo. ${ }^{(2)}$ En Uruguay particularmente, la Periodontitis alcanza al $30 \%$ de la población mayor de 35 años. ${ }^{(3)}$ La misma afecta no solo a la cavidad oral pudiendo llevar a la pérdida dentaria, sino que también está relacionada con otras enfermedades sistémicas, como la diabetes, enfermedades cardiovasculares, obesidad, entre otros. ${ }^{(4-6)}$

Para una mejor comprensión de la enfermedad, es necesario realizar un abordaje integral de los distintos componentes del proceso. El interés de producir conocimiento desde una perspectiva integral, e identificar factores psico-sociales de la enfermedad periodontal ha sido el objetivo del estudio que sustenta el presente artículo. Es de señalar, que los estudios odontológicos tienen un corte biomédico y ha sido desde ese enfoque que se han investigado las enfermedades periodontales, así como también su abordaje en la atención. La búsqueda bibliográfica realizada da cuenta que existe un vacío en estudios odontológicos que aborden la enfermedad periodontal desde la perspectiva psicosocial-de los pacientes; visibilizando la carencia de estudios cualitativos al respecto. ${ }^{(7)}$ Hasta el momento no se identifican aún, estudios de la población del Uruguay que investiguen las implicancias subjetivas (pensamientos, afectos, comportamientos, etc.) en esta población.

Para poder analizar los aspectos ya mencionados que inciden en las personas que padecen este tipo de enfermedad es necesario integrar aportes de otras disciplinas. En ese sentido, la Psicología de la Salud contribuye a la comprensión de estos factores que se despliegan en entidades patológicas como la enfermedad periodontal.
Los factores psicológicos juegan un papel importante en la etiología de diversas enfermedades. No se le puede adjudicar a un factor psicológico la enfermedad con seguridad, pero se pueden prevenir enfermedades actuando en ellos. ${ }^{(8)}$

Elter plantea además que si el paciente posee un problema de salud mental contribuye a una progresión más rápida de la enfermedad periodontal, a través de la disminución de prácticas de autocuidado, cambios en la dieta, aumento del tabaquismo, además de favorecer otras conductas perjudiciales cómo el bruxismo. ${ }^{(9)}$

Las emociones también pueden afectar la respuesta inmune y endócrina; lo que hace que el individuo sea más susceptible al desarrollo de enfermedades psicosomáticas, aislamiento social, estados depresivos y ansiosos, etc. En la medida que la persona sienta que la enfermedad la desborda y no la puede controlar, surge el stress como expresión de ello. ${ }^{(10)}$

Las enfermedades crónicas introducen al sujeto en la incertidumbre, a la dependencia, al dolor, limitaciones funcionales y emocionales que impactan negativamente en él (tristeza, miedo, ansiedad y enojo). ${ }^{(11)}$

A nivel subjetivo la enfermedad crónica conlleva un proceso de duelo, ya que para el paciente es "un antes y un después" en su vida. El duelo implica enfrentar la pérdida de quien era antes de la enfermedad y que nunca más volverá a ser y por quien ya no se podrá ser. ${ }^{(11-12)}$

Martín Alfonso expresa que en todas las patologías subyace un conjunto de atributos que surgen de la valoración socio-cultural e histórica de las mismas. Estos influyen en la relevancia que poseen los síntomas, el miedo que genera, el componente agresivo de la misma y el desarrollo de las enfermedades. ${ }^{(13)}$

Los individuos están dentro de un entorno social que es complejo y dinámico. ${ }^{(14-15)}$ Del mismo modo, los seres humanos tienen necesidades que sólo pueden ser satisfechas a través de la interacción con otros. Es vital el reconocimiento y la estima por parte de otras personas. ${ }^{\left({ }^{(8)}\right.}$ 
Esta última cita lleva a señalar que algunas enfermedades además poseen una carga de estigma social que producen actitudes y actos de rechazo u omisión hacia las personas que la padecen. (16) Según Goffman, son atributos que se valoran negativamente, lo que generan en las personas sentimientos de vergüenza y humillación. ${ }^{16}$ En tal sentido, el estigma social tiene efectos negativos en el estado de salud, en la calidad de vida y en el proceso de algunas enfermedades. ${ }^{(16)}$

Los pacientes con enfermedad periodontal tienen sentimientos de vergüenza y culpa debido a su estado de salud bucal. ${ }^{(17)}$ La falta de piezas dentales puede repercutir de forma negativa en la autoestima del paciente, en su confianza y en el vínculo con su entorno (alteraciones laborales, en las relaciones interpersonales, escolares, entre otras). En la medida de que el paciente considere que su sonrisa no es estéticamente aceptable, puede influir en su modo de comportamiento frente a los demás (evitar el contacto social, apatía, desidia, etc.) y en el cuidado que se brinda a sí mismo. ${ }^{(17)}$

La enfermedad periodontal podría de este modo, afectar la calidad de vida de quienes la padecen. ${ }^{(7)}$

Desde la década de los 60 y 70 del siglo XX, el término calidad de vida es utilizado en las ciencias biomédicas y está vinculado con la salud, con las terapéuticas utilizadas y los resultados de los tratamientos. ${ }^{(18-19)}$ Se atribuye este vínculo a la creciente necesidad de obtener datos por parte de los pacientes, conocer sus experiencias, satisfacción y preocupaciones. ${ }^{(18-19)}$

En el actual milenio, a partir de los aportes de otras ciencias de la salud y sociales, la calidad de vida se define a partir de dimensiones objetivas y subjetivas. La dimensión objetiva se la puede determinar por factores externos como son los económicos, el socio-político, los culturales que favorecen u obstaculizan el desarrollo humano de las personas. La dimensión subjetiva se expresa a través de la valoración que los individuos realizan de su propia vida. ${ }^{(20)}$

Walker propone que esta dimensión subjetiva del constructo se relaciona con el bienestar, satisfacción con la vida, auto reporte de salud y estado de salud física y mental. ${ }^{(21)}$

\section{Materiales y métodos}

El diseño de este estudio fue aprobado por el Comité de Ética de la Facultad de Odontología de la Universidad de la República (UdelaR) (Exp.091900-000116-19) y notificado al Comité de Ética de la Facultad de Psicología de la UdelaR (2019), contemplando principios éticos y bioéticos de diferentes Declaraciones; dentro de ellas la Declaración de Helsinki. ${ }^{(22)}$

Se trató de un diseño metodológico mixto cuanti- cualitativo, a partir de la aplicación de una entrevista (semiestructurada) con base en la teoría fundamentada y el cuestionario OHIP14 (Oral Health Impact Profile). ${ }^{(23)}$

En el caso de las entrevistas, se grabaron digitalmente y se transcribieron con el consentimiento de los participantes.

La muestra fue seleccionada a partir de una lista de pacientes que fueron atendidos en la clínica de Periodoncia en la facultad de odontología UdelaR en el período de marzo 2017-diciembre 2019. Los criterios de inclusión para la muestra correspondieron a pacientes adultos que fueron atendidos en la clínica de periodoncia de la Facultad de Odontología (UdelaR). Los criterios de exclusión fueron pacientes con enfermedades psiquiátricas o cualquier otra enfermedad que les impida el correcto entendimiento de la información que se les brinda. El trabajo de campo fue realizado por un solo operador AA. El tamaño de la muestra se adecuó al criterio de la saturación de la información que emergió del análisis de las entrevistas.

Se efectuó un análisis interpretativo del discurso de los pacientes. 
Para el proceso de interpretación, se utilizó el $\mathrm{MaxQDA}^{\oplus}$ cómo herramientas tecnológica y apoyo en la categorización, análisis y triangulación de la información obtenida.

Las preguntas de la entrevista se construyeron en base a la literatura sobre Psicología de la Salud y sobre enfermedad periodontal. (18; 24-26) La pauta de entrevista constó de preguntas con un lenguaje accesible para su comprensión y se tuvo en cuenta la necesidad de generar un clima que permitiera que el participante se sintiera cómodo para responder.

Se realizaron memorandos con las percepciones del investigador, registrando las reacciones de los participantes y cuándo el estado emocional se traducía en llanto o en otras manifestaciones emocionales.

Se realizó un procedimiento analítico para construir teoría que se fundamentó en los datos (Teoría fundamentada). A partir de ellos se desarrollaron las conceptualizaciones. ${ }^{(27)}$

La aplicación del cuestionario validado de perfil de impacto en salud oral OHIP-14, está integrado por 14 preguntas, las que permitieron evaluar la frecuencia con que una persona experimenta dificultades para cumplir determinadas funciones y cómo repercuten o no, sus problemas de salud bucal en la calidad de vida diaria. ${ }^{(23 ; 28-29)}$ Cada pregunta tiene un valor que va del 0 al 4 . Para su análisis se utilizó el programa RStudio versión 4.0.3 para Windows, el tipo de análisis estadístico fue realizado para cada variable señalada en esta investigación de forma individual utilizando los paquetes Rcmdr y sjPlot.

El cuestionario relevó datos demográficos que contribuyeron a la caracterización de los participantes. Se estudiaron las variables: edad, sexo al nacimiento, etnia, lugar de atención odontológica actual y nivel de instrucción.

\section{Análisis de los resultados}

Participaron 21 sujetos, 52,4\% mujeres y 47,6\% hombres.

El rango de edades fue entre 25 y 66 años, siendo el promedio de edad 49, 57 años (desvió estándar de 11, 37 años). El 14,3\% fueron menores de 40 ańos. El 8,1\% se encontraban entre la franja etaria de 40 a 49 ańos. El 23,8\% entre 50 y 59 años y el 23,8\% restante, mayores de 60 ańos de edad. El 90,5\% de los participantes se identificaron con el grupo étnico blancos o mestizos, mientras que el 9,5\% declararon ser afrodescendientes.

En cuanto a la variable nivel de instrucción, el 23,8\% señaló tener primaria completa, el 19\% realizó hasta ciclo básico, el 42,9\% tenía secundaria completa, $14,3 \%$ había alcanzado estudios terciarios sin completar. El 81\% de los participantes indicó que la atención de su salud bucal lo realizaba en la Facultad de Odontología de la UdelaR exclusivamente y el 19\% lo realiza en Facultad y en servicios privados.

Del discurso de los entrevistados se identificaron los aspectos subjetivos que repercutieron en mayor parte en ellos:

- afectación emocional tras el diagnóstico de la enfermedad periodontal como tristeza, rabia y miedo.

- afectación a nivel social donde la persona se recluye y evita el contacto con los demás.

- sentimientos de vergüenza, rechazo a ellos mismos y baja autoestima.

La afectación emocional tuvo una frecuencia del $81 \%$ en las respuestas dadas por los participantes. Por otro lado, la repercusión a nivel social se expresa con una frecuencia del $62 \%$ en las respuestas y los sentimientos de vergüenza, rechazo y/o baja autoestima son mencionados en un $28,5 \%$. (Tabla 1 ) 
Tabla 1: Aspectos subjetivos que se presentaron con mayor frecuencia en las entrevistas

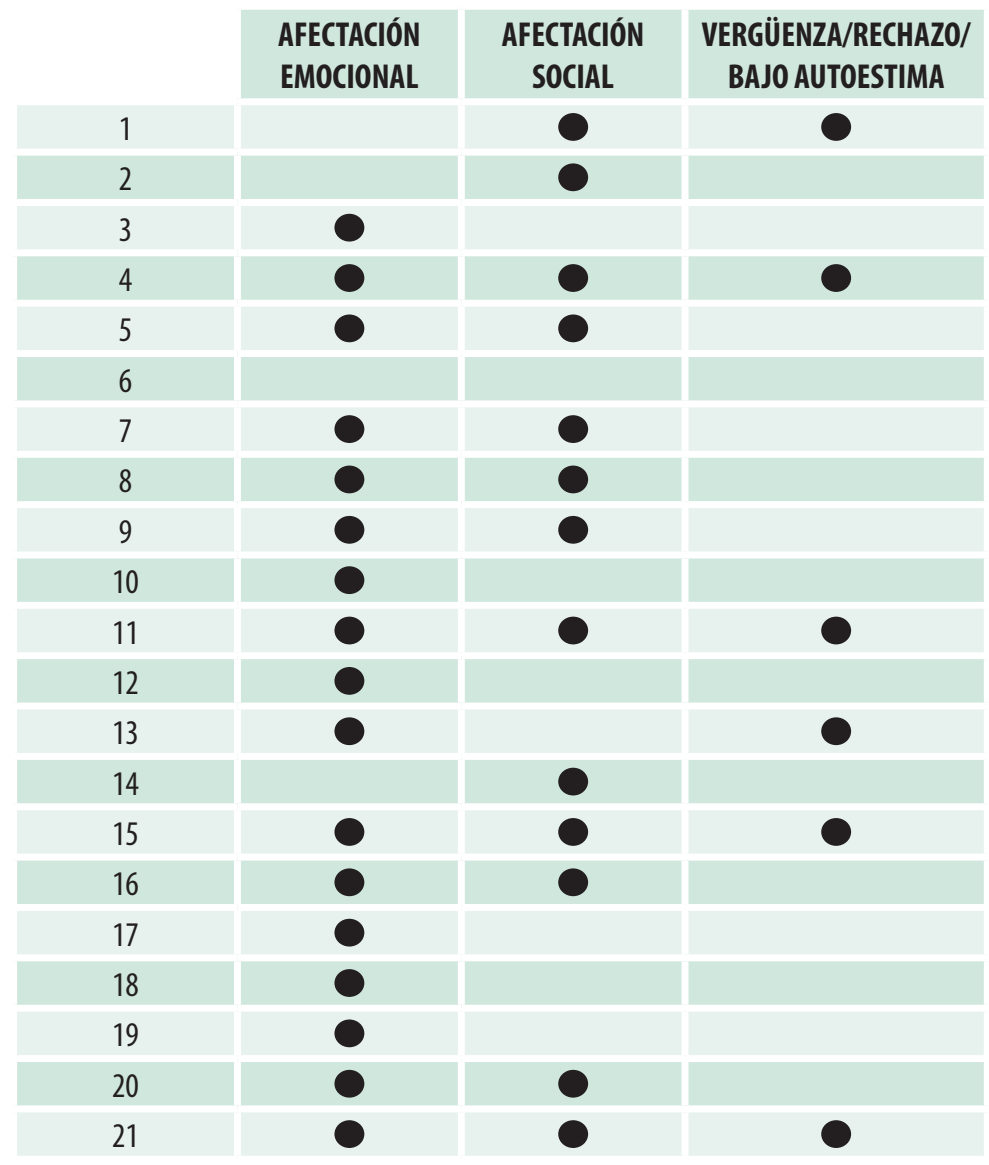

Frente a la pregunta "¿Qué sintió y/o pensó cuando le diagnosticaron la enfermedad periodontal?", algunas de las respuestas obtenidas fueron:

... "me dio tristeza, me senti depresiva..." (mujer, 33 años)

... "me dio rechazo a mi misma” ... (mujer, 42 años)

... "me dio mucha rabia, me siento frustrada..." (mujer, 33 años)

... "te sentis disminuido, es una sensación feisima..." (hombre 65 años)

Los participantes transitaron por estados de depresión y tristeza.

... "sali llorando, me quería morir" ... (mujer, 48 años)
... "no quiero saber de la enfermedad porque me puedo deprimir" ... (mujer, 56 años)

Del mismo modo, los participantes expresaron que la enfermedad periodontal repercutió en sus vínculos y en el relacionamiento social. En algunos provocó aislamiento y baja autoestima. En otros, sentimientos de culpa y enojo debido a la enfermedad.

... "me quedó la autoestima por el piso porque los dientes son tu presentación..." (mujer 53 años)

... "si hablas con alguien te siente el mal aliento, se te cierran todas las puertas..." (hombre, 48 años) ... "me da vergüenza reirme"... (mujer, 33 años)

Los participantes se sienten estigmatizados, discriminados y poco comprendidos por los otros. Del mismo modo, plantean que la enfermedad 
conlleva desventajas a nivel social. Lo que se traduce en aislamiento y distanciamiento social. ... "me excluyo de las reuniones y de muchas cosas por no tener bien mis dientes"... (hombre 66 años) ... "me causó la separación con mi novia, ella nunca va a entender que es esta enfermedad y que me generó"... (hombre, 49 ańos)

Esta población evita sonreír. Aluden tener mayores dificultades en el acceso al mercado laboral debido a la enfermedad periodontal.

Los participantes del estudio expresaron preocupación, temor, miedo y tristeza de perder sus piezas dentarias y las repercusiones que eso pueda traer en sus vidas.

En cuanto a la calidad de vida, el cuestionario OHIP-14 brindó la siguiente información: los participantes presentaron un nivel de afectación en la calidad de vida de 1,46 (desvío estándar de 1,43 ) en promedio (Fig. 1), siendo la puntuación mínima de 0,38 y la máxima de 2,90 (Tabla 2). La escala va de 0 al 4 , donde 4 es la máxima afectación, significando que existe afectación en la calidad de vida de los pacientes con enfermedad periodontal.

Fig. 1: Valor medio de la pregunta (DE=Desvío Estándar)

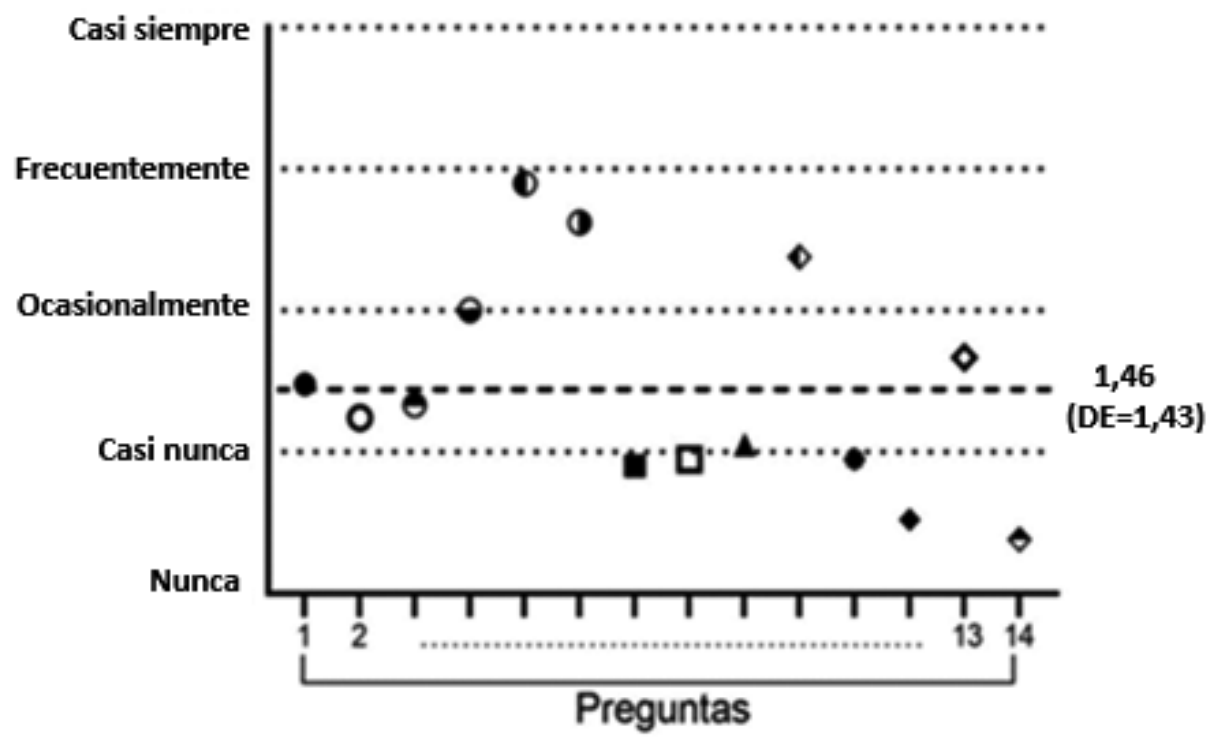

Tabla 2: Respuesta media y desvío estándar (D.E.) por participante

\begin{tabular}{|c|c|c|c|c|c|c|c|c|c|c|c|c|c|c|}
\hline & P. 1 & P. 2 & P. 3 & P. 4 & P. 5 & P. 6 & P. 7 & P. 8 & P. 9 & P. 10 & P- 11 & P- 12 & P. 13 & P. 14 \\
\hline Media & 1,48 & 1,24 & 1,33 & 2.00 & 2,90 & 2.62 & 0,90 & 0.95 & 1,05 & 2,38 & 0,95 & 0.52 & 1,67 & 0.38 \\
\hline D.E. & 1.54 & 1.30 & 1.35 & 1.34 & 1.00 & 1.16 & 1.22 & 1.24 & 1.40 & 1.36 & 1.24 & 1.03 & 1.39 & 0.74 \\
\hline
\end{tabular}

Las participantes femeninas señalaron un mayor nivel de afectación en la calidad de vida que los participantes varones (Fig. 2). 
Fig. 2: Afectación según sexo, $F=$ femenino, $M=$ masculino (D.E.=Desvío Estándar)

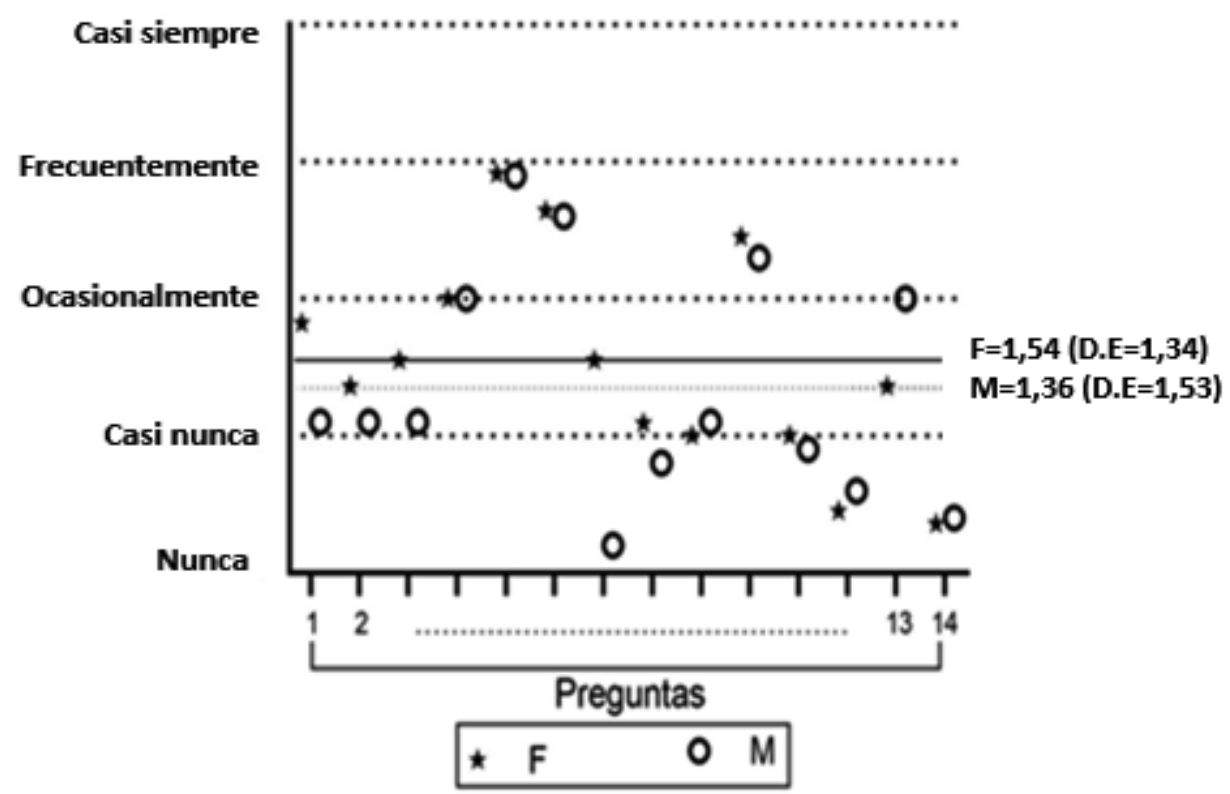

La variable edad no tuvo incidencia en el nivel de afectación de la calidad de vida (Fig. 3).

Fig. 3: Media por grupo etario (D.E.=Desvío Estándar)

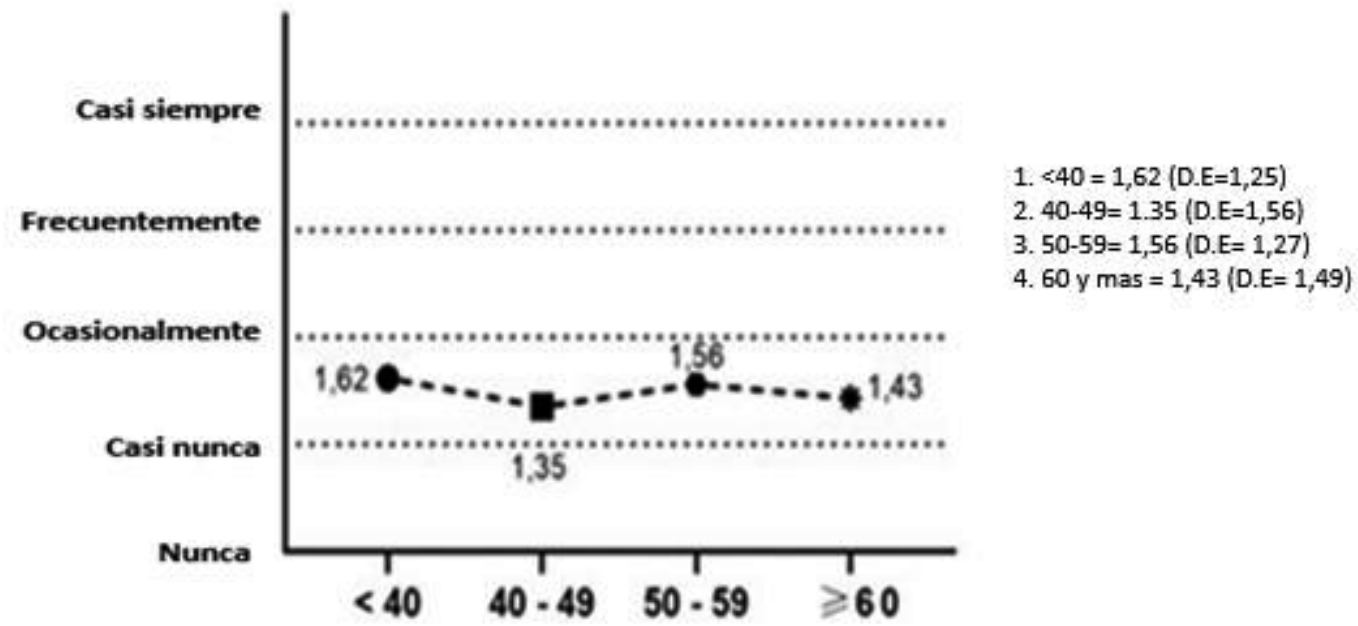

En cuanto a la variable nivel educativo se observa que a mayor grado de instrucción la afectación es levemente mayor. (Fig. 4) 
Fig. 4: Afectación según nivel de instrucción (D.E.=Desvío Estándar)

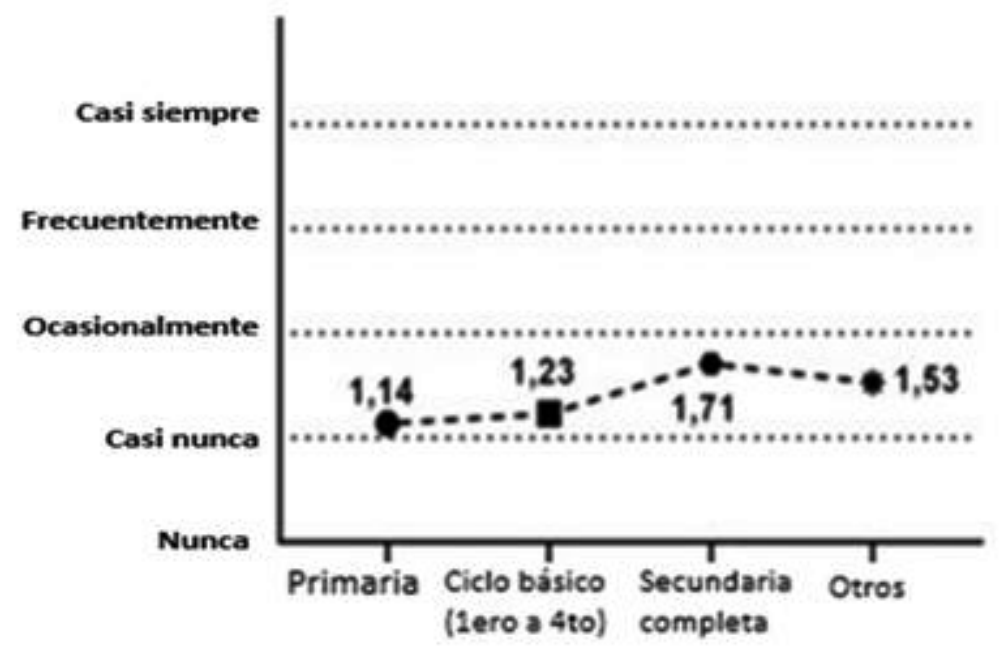

Primaria $=1,14$ (D.E=1,56)

Ciclo básico $=1,23$ (D.E $=1,28$ )

Secundaria $=1,71$ (D.E $=1,29)$

Otros $=1,53$ (D.E $=1,64)$

Otros son tecnicaturas y estudios universitarios sin completar.

\section{Discusión}

Los resultados de la investigación desarrollada tienen coincidencia con las conclusiones que llegan otras investigaciones a nivel internacional. $(8,10,1517,30)$ La población estudiada evidencia que el factor emocional y social posee una relevancia significativa en la enfermedad periodontal por la frecuencia que surge en las respuestas. ${ }^{(10,17)}$

La desmotivación, la baja autoestima, sentimientos de vergüenza y discriminación, surgen con relevancia en el discurso de los participantes. Sus pensamientos y sentimientos se ven afectados en diferentes grados dependiendo de cada participante. Evitan sonreír con lo que implica para el contacto y el afecto con los otros. En nuestra sociedad la mirada como la sonrisa son atributos importantes en el encuentro con el otro. A través de ellas, damos cuenta de nuestros estados de ánimo, nuestra apertura a relacionarnos, etc. Se rechazan a ellos mismos y se sienten limitados en el establecimiento de vínculos y en su socialización. La evidencia refiere que la salud oral es un componente significativo en la calidad de vida de estas personas con re- percusiones en su estado de salud general. En concordancia con la literatura, la aprobación y la estima de los demás son factores importantes para la salud mental. ${ }^{(8)}$

Los participantes manifiestan tener depresión. Según estudios, la depresión causada por esta enfermedad, tienen una consecuencia negativa en los resultados del tratamiento periodontal. ${ }^{(9)}$ La depresión agrava la enfermedad periodontal reduciendo la función del sistema inmunitario y produciendo un estado de inflamación. ${ }^{(10)}$

En adición a lo anteriormente mencionando, en la literatura se muestra que la depresión puede aumentar los malos comportamientos de salud, empeorar la higiene bucal, fumar o beber, una dieta poco saludable y sedentarismo. ${ }^{(25)}$

La enfermedad cobra un lugar relevante que los limita en desarrollar otras áreas de su vida como son la laboral, educativa y social. Su imagen personal es negativa. Se sienten incompetentes y sin confianza para enfrentar los problemas de la vida cotidiana.

La incidencia de factores psicológicos tiene influencia en el proceso de salud-enfermedad de los pacientes. ${ }^{(25)}$ 
Es de señalar que solo un participante expresó que la enfermedad no tuvo repercusiones personales.

Las participantes femeninas con enfermedad periodontal se ven más afectadas que los varones en su calidad de vida. Esto coincide con la literatura que estudia la afectación en la calidad de vida según género para otras enfermedades. (31-32) Esto último se puede relacionar con los prototipos estéticos de la sociedad contemporánea. Las mujeres son las más exigidas en lograr una estética acorde con los cánones de belleza que se han definido socialmente para tener éxito en el marcado laboral y en su vida personal. Dentro de los cánones de belleza, una sonrisa armoniosa, una dentadura perfecta, hace que las personas que padecen enfermedad periodontal, se sientan desvalorizadas. Las mujeres que participaron del estudio expresan rechazarse a sí mismas (autopercepción negativa) por no lograr alcanzar la imagen deseada, lo que es referido por Muñiz. ${ }^{(33)}$

Del mismo modo, quedó demostrado en esta población estudiada que, a mayor nivel de instrucción, es mayor la afectación en la calidad de vida a causa de la enfermedad periodontal. Es de señalar, que no se encontró en la literatura una asociación directa entre estas variables. Una hipótesis es que, si se tiene en cuenta que la educación es un factor de movilidad social y de integración laboral, las personas con mayor instrucción tienen un grado de mayor conciencia de la relevancia de la apariencia personal. Es uno de los requisitos que se pueden plantear en algunas ofertas del mercado laboral. En una sociedad mercantilista la estética se asocia a una jerarquía social para lograr éxito en la vida y tener mayor competitividad laboral. ${ }^{34)}$

El componente cuantitativo del estudio no permitió encontrar grandes diferencias en el nivel de afectación en las distintas franjas etarias. Esto puede obedecer a que la muestra fue pequeña como para generalizar los resultados. Sin embargo, hay una leve tendencia en los menores de 40 años a tener mayor afectación. En este punto se encuentra una coincidencia con la literatura consultada. ${ }^{(35-36)}$

Slade alude que a mayor edad las personas tienden a aceptar el deterioro en la salud con más naturalidad y en el caso de los más jóvenes las expectativas son más altas con respecto a la salud. ${ }^{(36)}$

\section{Conclusiones}

Este estudio demostró que la enfermedad periodontal genera un impacto biopsicosocial y en la calidad de vida de la población relevada. Se evidenció la afectación emocional tras el diagnóstico de la enfermedad.

Es necesario que los odontólogos tengan una mirada integral de sus pacientes y la incidencia de diferentes factores en la enfermedad periodontales. El estilo de vida, los hábitos alimenticios, el autocuidado, las concepciones de saludenfermedad, el contexto socio-cultural, entre otros, pueden estar determinando el éxito o no del tratamiento y la calidad de vida de los pacientes. Todo profesional de la salud tiene que desplegar en la atención a los pacientes su capacidad de empatía, de escucha y una buena comunicación para identificar las necesidades de sus pacientes. Por otra parte, los resultados de la investigación permiten proponer la importancia de desarrollar un abordaje de la enfermedad periodontal desde una perspectiva interdisciplinar para lograr una atención integral. Sobre todo, si se tiene en cuenta las diferentes entidades patológicas concomitantes que se pueden asociar y la repercusión en la calidad de vida de las personas.

Se sugieren futuras líneas de investigación que retomen este trabajo, ampliando el número de participantes y tomando otros contextos socio culturales de nuestro país. Si tomamos en cuenta que el contexto socio económico, etnia, sexo al nacimiento, edad y el nivel educativo se asocian con la ocurrencia de la enfermedad. ${ }^{(37)}$ Un mayor número de participantes nos permitiría estudiar mejor estas variables y su incidencia en la calidad de vida. 
Cómo reflexión final se destaca la importancia de incluir en los estudios epidemiológicos de periodoncia a los factores psico sociales. Se deben realizar más investigaciones en las comunidades que nos permitan validar instrumentos para medir estos factores.

Conocer las necesidades de cada comunidad permitirá mejorar las políticas públicas.

Una forma de implementar el trabajo multidisciplinarios es que los psicólogos formen parte de las clínicas odontológicas. Otra manera sería incorporando en la práctica clínica cuestionarios validados para poder detectar los factores psicosociales. Esto permitiría ampliar el enfo- que clínico y se podría incluir en el plan de tratamiento periodontal si es necesario, por ejemplo, técnicas para disminuir el estrés.

Si se abordan los factores psicosociales estamos también abordando la causa de la enfermedad, teniendo en cuenta que estos factores determinan las conductas del individuo. De esta forma estamos haciendo promoción de salud.

\section{Agradecimientos}

Empresa DENTAID, Roberto Volfovicz y Maité Souyet.

\section{Referencias}

1. Papapanou PN, Sanz M, Buduneli N, Dietrich T, Feres M, Fine DH, Flemmig TF, Garcia R, Giannobile WV, Graziani F, Greenwell H, Herrera D, Kao RT, Kebschull M, Kinane DF, Kirkwood KL, Kocher T, Kornman KS, Kumar PS, Loos BG, Machtei E, Meng H, Mombelli A, Needleman I, Offenbacher S, Seymour GJ, Teles R, Tonetti MS. Periodontitis: Consensus reporto of Workgroup 2 of the 2017 World Workshop on the Classification of Periodontal and Peri-Implant Disesases and Conditions. J Clin Periodontol 2018; 45(20): 162-170.

2. Pihlstrom BL, Michalowicz BS, Johnson NW. Periodontal diseases. The Lancet. 2005; 366(9499): 1809-20.

3. Lorenzo S, Alvarez R, Andrade E, Piccardo V, Francia A, Massa F, Correa MB, Peres MA. Periodontal conditions and associated factors among adults and the elderly: findings from the first National Oral Health Survey in Uruguay. Cad Saúde Pública. 2015; 31(11): 242536.

4. Graves D, Zhenjiang D, Yingming Y. The impact of diabetes on periodontal diseases. Periodontology 2000 2020; 82: 214-224.

5. Carrizales E, Ordaz A, Vera R, Flores R. Periodontal disease, systemic inflammation and the risk of cardiovascular disease. Heart Lung Circ. 2018 Nov; 27(11): 1327-1334.

6. Martinez M 1, Silvestre J, Silvestre F. Association between obesity and periodontal disease. A systematic review of epidemiological studies and controlled clinical trials. Med Oral Patol Oral Cir Bucal. 2017; 22 (6): 708-15.

7. Wong L, Yap A, Allen P. Periodontal disease and quality of life: Umbrella review of systematic reviews. J. Periodont. Res.. 2020; 00: 1-17 10.1111/jre.12805.

8. Kaplan BH, Cassel JC, Gore S. Social support and health. Med Care. 1997; 15: 47-58

9. Elter J, White A, Gaynes B, Bader J. Relationship of Clinical Depression to Periodontal Treatment Outcome. J Periodontol. 2002; 73: 441-449.

10. Warren KR, Postolache TT, Groer ME, Pinjari O, Kelly DL, Reynolds MA. Role of chronic stress and depression in periodontal diseases. Periodontol 2000. 2014; 64(1): 127-38.

11. Dapueto J, Varela B. Modelos y praxis psicológicos en la medicina. Montevideo: Psicología Médica, 2016

12. Tizón García JL. Componentes psicológicos de la práctica médica, una perspectiva desde la atención primaria. Barcelona: Doyma, 1988. 272 p. 
13. Martín Alfonso L. Aplicaciones de la psicología en el proceso salud enfermedad. Rev Cub Salud Pública 2003; 29(3): 275-281.

14. Peruzzo DC, Benatti BB, Ambrosano GM, Nogueira-Filho GR, Sallum EA, Casati MZ, Nociti FH Jr. A systematic review of stress and psychological factors as possible risk factors for periodontal disease. J Periodontol 2007; 78: 1491-1504.

15. Moss ME. Exploratory case-control analysis of psychosocial factors and adult periodontitis. J Periodontol. 1996; 67(10): 1060-9.

16. Goffman E. Estigma. La identidad deteriorada. Buenos Aires: Amorrortu, 1963.

17. Abrahamsson K, Wennströma J, Hallbergc U. Patients' Views on Periodontal Disease; Attitudes to Oral Health and Expectancy of Periodontal Treatment: A Qualitative Interview Study. Oral Health Prev Dent 2008; 6: 209-216.

18. Locker D, Allen F. What do measures of "oral health-related quality of life" measure? Community Dent Oral Epidemiol. 2007 Dec; 35(6): 401-11.

19. Meeberg GA. Quality of life: A concept analysis. J Adv Nurs 1993; 18: 32- 38.

20. García-Viniegras C, González Benítez I. La categoría bienestar psicológico. Su relación con otras categorías sociales. Rev Cubana Med Gen Integr 2000; 16(6): 586-92.

21. Walker A. Understanding Quality Of Life in Old Age. Berkshire England: Open University Press, 2005.

22. Declaración de Helsinki de la Asociación Médica Mundial. Gac Med Mex 2001; 137(4): 387-390.

23. Montero J, Bravo M, Albaladejo A, Hernández L, Rosel E. Validation the oral health impact profile (OHIP-14sp) for adults in Spain. Med.Oral Patol Oral Cir Bucal 2009; 14(1): 44-50

24. Decker A, Askar H, Tattan M, Taichman R, Wang HL. The assessment of stress, depression, and inflammation as a collective risk factor for periodontal diseases: a systematic review. Clin Oral Investing 2020; 24(1): 1-12.

25. Lacopino A. Relationship between stress, depression and periodontal disease J Can Dent Assoc 2009 Jun; 75(5): 329-30.

26. Morales Calatayud F. Psicología de la salud. Realizaciones e interrogantes tras cuatro décadas de desarrollo. Rev. Latinoamericana de Ciencia Psicológica. 2012; 4(2): 98-104.

27. Strauss A, Corbin J. Grounded theory methodology: An overview. En: N. K. Denzin \& Y. S. Lincoln (Eds.), Handbook of qualitative research 1994; p. 273-285.

28. Slade GD. Derivation and validation of a short-fortn oral health impact profile. Community Dent Oral Epidemiol 1997; 25(4): 284-90.

29. Slade GD. Measuring Oral Health and Quality of Life.: Chapel Hill: University of North Carolina. Department of Dental Ecology School of Dentistry. 1997 Disponible en: https: //www.adelaide.edu. $\mathrm{au} /$ arcpoh/downloads/publications/reports/miscellaneous/measuring-oral-health-and-quality-of-life. pdf

30. Arrivillaga M, Salazar I, Gómez I. Prácticas, creencias y factores del contexto relacionados con estilos de vida de jóvenes y adultos. Pontificia Universidad Javeriana. Psicología Conductual. 2005; 13(1): 19-36.

31. Cavallo F, Zambon A, Borraccino A, Raven-Sieberer U, Torsheim T, Lemma P. HBSC Positive Health Group. Girls growing through adolescence have a higher risk of poor health. Qual Life Res 2006; 15: 1577-85.

32. Serra-Sutton V, Rajmil L, Aymerich M. Estrada MD. Desigualtats de genero en la percepció de la salut durant l'adolescencia. Annals de Medicina. 2004; 87: 25-9.

33. Muñiz E. Pensar el cuerpo de las mujeres: cuerpo, belleza y feminidad. Una necesaria miranda feminista. Soc. Estado 2014; 29(2). Disponible en: https: //doi.org/10.1590/S0102-69922014000200006

34. Solano F, Ortiz V. La estetización del mercado laboral: modelos estéticos demandados por el trabajo en las sociedades contemporáneas. Antropol. Sociol. 2015; 17(2): 15-36.

35. León S, Bravo-Cavicchioli D, Correa-Beltrán G, Giacaman R. Validation of the spanish version of the Oral Health Impact Profile (OHIP-14Sp) in elderly Chileans. BMC Oral Health. 2014; 14: 95. 
36. Slade GD, Sanders AE. The paradox of better subjective oral health in older age. J Dent Res 2011; 90 (11): 1279-1285.

37. Oppermann R, Hass A, Kuchenbecker C, Susin C. Epidemiology of periodontal diseases in adults from Latin America. Perodontol.2000 2015; 67(1): 13-33.

\section{Nota declaración de intereses:}

Los autores declaran no tener conflicto de intereses. Esta investigación no fue subsidiada por ninguna empresa o persona en particular.

\section{Nota contribución de los autores:}

1. Concepción y diseño del estudio

2. Adquisición de datos

3. Análisis de datos

4. Discusión de los resultados

5. Redacción del manuscrito

6. Aprobación de la versión final del manuscrito

AAriceta ha contribuido en: 1, 2, 4, 5 y 6 .

LB ha contribuido en: 1 y 6 .

AArias ha contribuido en: 3, 4, 5 y 6 .

EAndrade ha contribuido en: 1 y 6 .

\section{Nota de aceptación:}

Este artículo fue aprobado por la editora de la revista Mag. Dra. Vanesa Pereira-Prado. 